\title{
Test de l'hypothèse démocratique dans les décisions budgétaires
}

\section{communales}

\author{
Monsieur Jean-Jacques Laffont, Monsieur Jacques Le Pottier, Monsieur Yves Aragon
}

\section{Résumé}

Test de l'hypothèse démocratique dans les décisions budgétaires communales

Le financement des dépenses communales peut être, jusqu'en 1980, assimilé à une décision unidimensionnelle concernant le nombre de centimes. Cet article propose une modélisation de ce choix par la règle majoritaire qui résulte en le meilleur choix de l'agent médian dans une population où l'on a distingué ceux qui payent et ceux qui ne payent pas la taxe professionnelle. Le modèle est estimé sur des données concernant l'agglomération toulousaine. L'hypothèse démocratique selon laquelle les deux catégories d'agents mentionnées ci-dessus ont le même poids dans les décisions communales est rejetée.

\section{Abstract}

Test of the democratic hypothesis in French communities

The financing of local public expenditures can be viewed, until 1980, as a one dimensional choice. This paper proposes a model to formalize this choice based on the majority rule. It results in the best choice of the median agent in a population in which two types of citizens have been distinguished, those who pay and those who do not pay the " professional tax ". The model is estimated using data about the region of Toulouse, France. The democratic hypothesis according to which both types of agents mentioned above have the same weight in the decisions is rejected.

\section{Citer ce document / Cite this document :}

Laffont Jean-Jacques, Le Pottier Jacques, Aragon Yves. Test de l'hypothèse démocratique dans les décisions budgétaires communales. In: Revue économique, volume 39, n², 1988. pp. 405-420;

http://www.persee.fr/doc/reco_0035-2764_1988_num_39_2_409070

Document généré le 29/05/2016 


\title{
Test de l'hypothèse démocratique dans les décisions budgétaires communales
}

\author{
Yves Aragon \\ Jean-Jacques Laffont \\ Jacques Le Pottier *
}

\begin{abstract}
Le financement des dépenses communales peut être, jusqu'en 1980, assimilé à une décision unidimensionnelle concernant le nombre de centimes. Cet article propose une modélisation de ce choix par la règle majoritaire qui résulte en le meilleur choix de l'agent médian dans une population où l'on a distingué ceux qui payent et ceux qui ne payent pas la taxe professionnelle. Le modèle est estimé sur des données concernant l'agglomération toulousaine. L'hypothèse démocratique selon laquelle les deux catégories d'agents mentionnées ci-dessus ont le même poids dans les décisions communales est rejetée.
\end{abstract}

Dans la pratique, les décisions concernant les biens publics locaux sont très complexes en raison des formes variées de financement et du nombre de biens concernés. Or la théorie politico-économique disponible pour des recherches empiriques se ramène essentiellement à la théorie de l'agent médian (Bergstrom et Goodman [1973], Romer, T. et H. Rosenthal [1979], Comanor [1976] ...) qui requiert à la fois l'unidimensionnalité de la décision et des préférences unimodales. Dans ce cas, le vote à la majorité simple conduit au meilleur choix de l'agent médian. Cette prédiction peut alors être confrontée aux données.

Il se trouve que les méthodes de financement des dépenses communales (jusqu'en 1980 pour toutes les communes, après 1980 pour certaines d'entre elles) peuvent être assimilées à une décision unidimensionnelle malgré leur complexité apparente. Bien que le financement propre de la commune soit assuré par quatre taxes (taxe d'habitation, impôt foncier bâti, impôt foncier non bâti, taxe professionnelle), la structure de ces

* Nous remercions pour leur collaboration précieuse dans la collecte des données MM. Lhomme de la Trésorerie générale de Toulouse, Vuillemin et Brunel des services fiscaux du département de la Haute-Garonne, Leddet de la direction générale des Impôts, et Gérard des Archives de la Haute-Garonne. Nous remercions également $\mathrm{E}$. Malinvaud et $\mathrm{C}$. Gourieroux et deux rapporteurs anonymes pour leurs remarques constructives. 
impôts est figée et une seule décision doit être prise concernant le niveau général de la taxe ${ }^{1}$ (le nombre de centimes). Si donc on veut bien agréger l'ensemble des dépenses communales en un bien public local, la décision collective est ramence au choix unidimensionnel du nombre de centimes.

Certes, les décisions ne sont pas prises comme dans certains pays par vote direct des habitants mais par le conseil municipal. On peut toutefois faire l'hypothèse que ce conseil représente de manière démocratique l'ensemble de la population de la commune. Si les fonctions objectifs des habitants sont unimodales dans le niveau des taxes, on peut alors faire la prévision que les décisions communales correspondent au meilleur choix de l'agent médian.

Dans cette note, nous poursuivons cette voie. Toutefois une complication inévitable doit être surmontée. Il faut pour le moins distinguer deux types d'agents dans la commune : ceux qui payent la taxe professionnelle et ceux qui ne la payent pas.

Dans la deuxième section, nous construisons un modèle de décision collective qui distingue ces deux types d'agents. Nous postulons des fonctions d'utilité directes conduisant à des fonctions d'utilité indirectes qui sont bien unimodales dans le niveau des taxes. Sous l'hypothèse démocratique (un habitant une voix), on est ainsi conduit à une équation déterminant le niveau de la taxe en fonction de paramètres à estimer et d'un certain nombre de variables exogènes. Etant donné la distinction très nette qui existe entre les deux types d'agents considérés, on peut se demander si de fait les agents qui payent la taxe professionnelle n'ont pas un poids relatif plus grand dans les décisions communales. On construit un modèle qui fait cette distinction pour pouvoir tester l'hypothèse démocratique.

La troisième section présente la méthode d'estimation, la quatrième les données. La section 5 présente les résultats d'estimation essentiels. Diverses variantes sont présentées à la section 6 . Quelques conclusions sont rassemblées à la section 7 .

\section{LE MODĖLE DE DÉCISION COLLECTIVE DANS LA COMMUNE}

Considérons une collectivité dont tous les agents ont le même type de fonction d'utilite :

$$
U(x, s, z)=\alpha \log x+\beta \log s+\gamma \log z
$$

1. Jusqu'en 1980 le conseil municipal vote uniquement le montant total des impäts à percevoir. Ce montant est ensuite réparti entre les quatre taxes en fonction de coefficients de répartition exogènes. A partir de 1981, les conseils municipaux votent directement les taux des quatre taxes. Certains d'entre eux cependant ont choisi de ne voter qu'un taux d'augmentation unique à appliquer aux taux des quatre taxes, ce qui conserve lunidimensionalité de la décision. 
où $s$ est le logement mesuré comme un agrégat de caractéristiques, notamment la surface et la localisation, $z$ est un agrégat représentant l'ensemble des biens publics fournis par la commune considérée et mesuré par le budget de la commune, $x$ est un agrégat de tous les biens consommés autres que le logement et les biens publics.

On prend $x$ comme numéraire et l'on note $r$ le prix du bien $s$.

Le budget de la commune s'écrit :

$$
z=h B_{h}+t B_{t p}+T
$$

où $h$ est le taux de la taxe d'habitation 1 dont la base est $B_{h}, t$ est le taux de la taxe professionnelle dont la base est $B_{t p}$ et $T$ est l'ensemble des autres recettes de la commune.

Nous définissons deux groupes d'agents, ceux qui payent la taxe professionnelle et ceux qui ne la payent pas.

Fonctions d'utilité indirectes des agents qui ne payent pas la taxe professionnelle

Nous allons définir une fonction d'utilité indirecte de l'agent type qui lui permet d'évaluer son bien-être comme fonction du taux de la taxe d'habitation lorsqu'il optimise son comportement sur les marchés.

Etant donné $h$ et $z$, l'agent type de revenu $\mathbf{R}$ résout le programme :

$$
\left\{\begin{array}{l}
\underset{(x, y)}{\operatorname{Max}}\{\alpha \log x+\beta \log s+\gamma \log z\} \\
\text { T.Q. } \quad x+(1+h) r s=\mathrm{R}
\end{array}\right.
$$

d'où

$$
\left\{\begin{array}{l}
s=\frac{\beta}{\alpha+\beta} \frac{\mathbf{R}}{(1+h)^{r}} \\
x=\frac{\alpha}{\alpha+\beta} \mathbf{R}
\end{array}\right.
$$

Notons $S$ la somme totale des surfaces occupées. La base de la taxe d'habitation est donc : $B_{h}=r S$.

D'après (1), $z=h r S+t B_{t p}+T$ que l'on peut écrire :

$$
z=h \mathbf{B}+\mathbf{T}
$$

1. Le modèle étant appliqué sur un ensemble de communes urbaines, l'exclusion de la taxe sur la propriété foncière non bâtie est légitime. La taxe sur la propriété foncière bâtie pose problème car elle n'est payée que par les propriétaires. Face à ce problème difficile nous adopterons deux attitudes extrêmes. Dans la première, nous négligerons cette taxe (variante $\mathrm{A}$ ), dans la seconde nous l'ajouterons à la taxe d'habitation en considérant qu'elle est en fait payée par tous les agents directement par les propriétaires, indirectement dans leur loyer par les locataires (variante B). 
où $B=\frac{h r S+t B_{t p}}{h}=\frac{\text { taxe d'habitation }+ \text { taxe professionnelle } 1}{h}$.

En substituant (3) et (4) dans la fonction d'utilité, nous obtenons :

$$
U^{*}(h)=-\beta \log (1+h)+\gamma \log (h B+T)+\text { Constante }
$$

Une condition suffisante pour que cette fonction soit unimodale est que $T / B<1$ (cela est vérifié par les données que nous avons utilisées). Sous cette hypothèse, la fonction $U^{*}(\cdot)$ atteint son maximum pour :

$$
h=\frac{\gamma-\frac{T}{B} \beta}{\beta-\gamma}
$$

Fonctions d'utilité indirectes des agents qui payent la taxe professionnelle

La modélisation précise de la taxe professionnelle n'est pas possible par manque de données. En effet, dans la pratique, la taxe professionnelle porte sur une base constituée à partir de la valeur locative des immobilisations d'une part, d'autre part, suivant les types d'agents, de la masse des salaires versés ou des recettes encaissées. Pour simplifier, nous supposons que la base de la taxe professionnelle est proportionnelle au revenu de l'agent considéré. Comme par ailleurs le taux de la taxe professionnelle et celui de la taxe d'habitation sont proportionnels, on peut écrire :

$$
t \mathrm{~B}_{t p}=h \delta \mathbf{R}
$$

Un agent type de revenu $R$ résout donc le programme suivant :

$$
\left\{\begin{array}{l}
\underset{(x, s)}{\operatorname{Max}}\{\alpha \log x+\beta \log s+\gamma \log z\} \\
\text { T.Q. } x+(1+h) r s=(1-\delta h) \mathbf{R}
\end{array}\right.
$$

d'où

$$
\left\{\begin{array}{l}
s=\frac{\beta}{\alpha+\beta} \frac{(1-\delta h) R}{(1+h) r} \\
x=\frac{\beta}{\alpha+\beta}(1-\delta h) R
\end{array}\right.
$$

En substituant (9) et (4) dans la fonction d'utilité, nous obtenons :

$\tilde{U}^{*}(h)=(\alpha+\beta) \log (1-\delta h)-\beta \log (1+h)+\gamma \log (B h+T)+$ Constante

1. B n'est pas fonction de $h$ car $B=r S+\frac{t}{h} B_{t, n}$ où $\frac{t}{h}$ est une constante (cf. note 1, p. 406). 
$\frac{\mathbf{T}}{\mathbf{B}}<1$ et $1-\delta \boldsymbol{h}>0$ sont des conditions suffisantes pour que la fonction $U^{*}(\cdot)$ soit unimodale en $h$ (cela est vérifié par les données que nous avons utilisées). Sous ces hypothèses, la fonction $\sigma^{*}(\cdot)$ atteint son maximum pour la valeur de $h$ solution de l'équation :

$$
-\frac{(\alpha+\beta) \delta}{1-\delta h}-\frac{\beta}{1+h}+\frac{\gamma}{h+\frac{T}{B}}=0
$$

Choix de $\mathrm{h}$

Puisque les préférences de tous les agents sont unimodales en $h$, le choix de $h$ par un vote à la majorité simple conduit au choix de $h^{*}$. qui correspond à l'agent médian.

Supposons que $\gamma$ est une fonction monotone du revenu $R$ de l'agent considéré, soit $\gamma(R)$. Affectons d'un indice 1 les agents qui ne payent pas la taxe professionnelle et d'un indice 2 ceux qui la payent. Pour chaque type d'agent il existe une relation biunivoque entre le revenu de l'agent et son niveau de taxe souhaité.

Pour un agent de type 1, d'après (6) :

$$
\mathbf{R}_{1}=\gamma^{-1}\left[\frac{\beta}{1+h_{1}}\left(h_{1}+\frac{T}{B}\right)\right]=\varphi\left(h_{1}\right)
$$

Pour un agent de type 2, d'après (11):

$$
\mathrm{R}_{2}=\gamma^{-1}\left[\left(\frac{(\alpha+\beta) \delta}{1-\delta h_{2}}+\frac{\beta}{1+h_{2}}\right)\left(h_{2}+\frac{T}{B}\right)\right]=\xi\left(h_{2}\right)
$$

Les fonctions $\varphi$ et $\xi$ sont monotones mais peuvent être simultanément croissantes ou décroissantes selon le type de monotonicité de $\gamma$. Ce choix sera laissé aux données.

Si on appelle $f_{1}$ et $f_{2}$ les fréquences des groupes d'agents de types 1 et 2 respectivement $\left(f_{1}+f_{2}=1\right)$ et $g_{1}(\mathbf{R}), g_{2}(R)$ les densités des distributions de revenu dans les deux groupes, $h^{*}$ est déterminé par l'équation :

$$
f_{1} f_{0}^{\cdot(n \cdot)} g_{1}(\mathrm{R}) d R+f_{2} f_{0}^{\xi(n \cdot)} g_{2}(\mathrm{R}) d R-\frac{1}{2}=0
$$

équation qui exprime que $h^{*}$ est choisi par l'agent médian.

Si l'on considère que les agents de type 1 n'ont pas le même poids dans la décision que les agents de type 2 (pour une voix d'un agent de type 1 un agent de type 2 a voix) $h^{*}$ est déterminé par l'équation :

$\frac{f_{1}}{f_{1}+v f_{2}} f_{0}^{\cdot(h \cdot)} g_{1}(R) d R+\frac{v f_{2}}{f_{1}+v f_{2}} f_{0}^{\xi(h \cdot)} g_{2}(R) d R-\frac{1}{2}=0$ 


\section{PROBLĖMES D'ESTIMATION ET DE TEST}

En donnant à la fonction $\gamma$ une forme fonctionnelle particulière, on peut chercher à estimer les paramètres $\alpha, \beta$ de la fonction d'utilité, les parametres de la fonction $\gamma(R)$ et le parametre $v$. Nous prenons $\boldsymbol{\gamma}(\mathbf{R})=e^{\mathbf{R}}$.

\section{Le modèle probabiliste}

Nous supposons que, pour chaque commune $k$, les distributions de revenu des deux sous-populations sont log-normales et sont caractérisées par les revenus moyens $\overline{\mathbf{R}}_{1 k}, \overline{\mathbf{R}}_{2 k}$ et les écarts types $\sigma_{1 k}, \sigma_{2 k}, k=1, \ldots, \mathbf{K}$.

Le modèle probabiliste à estimer s'écrit alors :

$\mathrm{B}\left(h^{*}{ }_{k},\left(\frac{\mathrm{T}}{\mathrm{B}}\right)_{k}, \delta_{k}, \overline{\mathrm{R}}_{1 k}, \overline{\mathrm{R}}_{2 k}, \sigma_{1 k}, \sigma_{2 k}, f_{1 k}, f_{2 k}, \alpha, \beta, a, v\right)=\varepsilon_{k} \quad k=1, \ldots, \mathrm{K}$

où $g$ représente la fonction non linéaire donnée en (15), $h^{*}{ }_{k}$ est la variable endogène.

$$
\left(\frac{T}{B}\right)_{k}, \delta_{k}, \overline{\mathbf{R}}_{1 k}, \overline{\mathbf{R}}_{2 k}, \sigma_{1 k}, \sigma_{2 k}, f_{1 k}, f_{2 k}
$$

sont les variables exogènes, $\alpha, \beta, a, v$ sont les paramètres à estimer, les $\varepsilon_{k}$ sont des variables aléatoires centrées indépendantes de variance $q_{k} \cdot \sigma^{2}$ où divers choix de $q_{k}>0$ pourront être faits pour prendre en compte une éventuelle hétéroscédasticité.

Sous l'hypothèse $v=1$, il faut utiliser l'équation (14) au lieu de (15).

Les méthodes d'estimation

a) Doubles moindres carrés non linéaires

Réécrivons l'équation (16) sous la forme compacte

$$
g\left(h^{*}{ }_{k} x_{k}, b\right)=\varepsilon_{k} \quad k=1, \ldots, \mathrm{K}
$$

où $x_{k}$ est un vecteur de variables exogènes et $b$ un vecteur de paramètres.

La méthode des doubles moindres carrés non linéaires de Amemiya [1975] consiste à minimiser :

$$
S_{k}(b / W)=g^{\prime} W^{\prime}\left(W^{\prime} W^{-1} W^{\prime} g\right.
$$


où $W$ est une matrice $(K, L)$ de constantes, de rang supérieur ou égal au nombre de paramètres à estimer.

L'estimateur ainsi obtenu $b$ est convergent et asymptotiquement normal. Soit $b_{0}$ le vecteur des vraies valeurs des parametres. Nous savons d'après Amemiya [1974] que :

$$
\sqrt{\mathbf{K}}\left(b-b_{0}\right) \stackrel{\mathscr{L}}{\rightarrow} \mathcal{N}\left(0, \sigma^{\alpha} \operatorname{plim} \mathrm{K}\left(\mathbf{G}_{0}^{\prime} \mathbf{P}_{\mathrm{w}} \mathbf{G}_{0}\right)^{-1}\right)
$$

où $\mathbf{P}_{\mathbf{w}}=\mathbf{W}\left(\mathbf{W}^{\prime} \mathbf{W}^{-1} \mathbf{W}^{\prime}\right.$

$$
G_{0}=\left(\frac{\partial G}{\partial b^{\circ}}\right)_{b_{0}}
$$

$\sigma_{2}$ peut être estimé par l'estimateur convergent

$$
\begin{gathered}
\hat{\sigma}^{a}=\frac{1}{\mathrm{~K}} \sum_{k=1}^{\mathrm{K}} g\left(h^{*} x_{k} x_{k}, b\right)^{2} \\
\left(\frac{\partial g}{\partial b^{\prime}}\right)_{b_{0}} \text { peut être estimé par }\left(\frac{\partial g}{\partial b^{\prime}}\right)_{\hat{b}}=\hat{G}
\end{gathered}
$$

La matrice des variances et covariances de $b$, qui permet de tester la significativité des paramètres, est alors :

$$
\sigma^{2}\left(\hat{G}^{\prime} P_{w} \hat{\mathbf{G}}\right)^{-1}
$$

\section{b) Le choix des meilleurs instruments}

Amemiya [1975] a montré que la matrice des variances et covariances asymptotique des doubles moindres carrés non linéaires était minimisée (au sens matriciel) par le choix $\mathrm{W}=\mathrm{EG}_{0}=\mathrm{E}\left(\frac{\partial g}{\partial b^{\prime}}\right)_{\mathrm{b}_{0}}$. Pour approximer ces instruments idéals mais non observables, Amemiya suggère d'utiliser un estimateur quelconque de la classe des doubles moindres carrés non linéaires, pour estimer $G_{0}$, par exemple $\hat{G}$ obtenu en $a$, et de chercher les variables exogènes qui président au mieux $\hat{G}$. Soit $W_{0}$ ce système d'instruments. Nous procéderons à une analyse canonique des deux ensembles de variables

$$
\left(\frac{\partial g}{\partial b^{\prime}}\right)_{b} \text { matrice }(K, L) \text {, d'une part }
$$

et

$$
\text { W, } \delta,\left(\frac{T}{B}\right), \overline{\mathbf{R}}_{1}, \overline{\mathbf{R}}_{2}, N_{1}, N_{2} \text { matrice }(K,(L+6)) \text {, d'autre part }
$$

où $\mathbf{W}$ est la matrice $(K, L)$ des instruments utilisés en $a$. Nous utilisons alors comme instruments les $\mathrm{L}$ premières composantes canoniques.

Soit $b^{*}$ l'estimateur ainsi obtenu. 


\section{c) Le maximum de vraisemblance}

Sous l'hypothèse additionnelle de normalité des erreurs $\varepsilon_{k}$, nous pouvons écrire la fonction de vraisemblance de notre modele et la maximiser en utilisant comme valeurs initiales les estimations obtenues en $a$ ou $b$.

Après maximisation par rapport à $\sigma^{2}$, nous obtenons le logarithme de la vraisemblance concentrée $L_{c}(b)$ (voir annexe) que l'on peut maximiser par rapport à $b$.

L'estimateur $\hat{b}$ ainsi obtenu est convergent, asymptotiquement normal et efficace et

$$
\sqrt{\mathbf{K}}\left(\hat{b}-b_{0}\right) \stackrel{\mathscr{L}}{\rightarrow} \mathscr{N}\left(0,-\left(\left.\lim \frac{1}{\mathrm{~K}} \frac{\partial^{\mathrm{s}} \mathrm{L}_{c}}{\partial \boldsymbol{b} \partial b^{\sigma}}\right|_{b_{0}}\right)^{-1}\right)
$$

où la matrice des variances et covariances asymptotique sera estimée par $\left(-\frac{1}{\mathbf{K}} \frac{\partial^{2} L_{c}}{\partial b \partial b^{\prime}} \mid \hat{b}\right)^{-1}$.

\section{LES DONNÉES}

Les données nécessaires pour estimer le modèle dans ses deux variantes $^{1}$ sont les suivantes, pour chaque observation $k$ :

1. les distributions du revenu dans chaque groupe 1 et 2 ;

2. les proportions $f_{1 k}$ et $f_{2 k}$ de chaque groupe;

3. la population des communes;

4. les taux $h^{*}{ }_{k}$ de la taxe d'habitation et $h^{\prime}{ }_{k}$ de la taxe d'habitation augmentée de l'impôt foncier sur les propriétés bâties ;

5. les rapports $\left(\frac{T}{B}\right)_{k}$ pour les variantes $\mathbf{A}$ et $\mathbf{B}$;

6. les coefficients $\delta_{k}$ pour les variantes $\mathbf{A}$ et $\mathbf{B}$.

Nous avons pu réunir ces données pour 51 observations correspondant aux 36 communes de l'agglomération toulousaine en 1980 et aux 15 communes de cette même agglomération qui, en 1981, ont fait varier proportionnellement les taux des quatre taxes.

1. Cf. note 1, p. 407 . 


\section{Les distributions du revenu}

Elles ont été obtenues à partir des sources fiscales. L'Administration fiscale nous a fourni :

a) pour la commune de Toulouse en 1980 et 1981 le nombre de foyers fiscaux par tranche de revenu et le revenu global de chaque tranche de revenu (treize tranches) pour les deux catégories de foyers fiscaux suivants :

- les foyers fiscaux dont aucun membre n'est imposé au titre des BIC ou BNC (sauf revenus accessoires). C'est la catégorie des foyers fiscaux qui ne payent pas la taxe professionnelle,

- les foyers fiscaux dont un membre au moins est imposé au titre des BIC ou BNC (pour des revenus non accessoires). C'est la catégorie des foyers fiscaux qui payent la taxe professionnelle.

Nous avons alors calculé les moyennes

$$
\overline{\mathbf{R}}_{1,36}^{80}, \overline{\mathbf{R}}_{2,36}^{80}, \overline{\mathbf{R}}_{1,36}^{81}, \overline{\mathbf{R}}_{2,36}^{81}
$$

et les variances

$$
\mathbf{V}_{1,36}^{80}, \mathbf{V}_{2,36}^{80}, \mathbf{V}_{1,36}^{81}, \mathbf{V}_{2,36}^{81}
$$

de ces distributions.

(36 est le numéro de la commune de Toulouse).

b) Pour les autres communes de l'agglomération toulousaine (35 communes), en 1980 et 1981 et pour chaque catégorie $i$ et $j:$ le revenu global et le nombre de foyers fiscaux.

Cela nous a permis de calculer les moyennes :

$$
\overline{\mathbf{R}}_{1, c}^{80}, \quad \overline{\mathbf{R}}_{2, c}^{80}, \quad \overline{\mathbf{R}}_{1, c}^{81}, \quad \overline{\mathbf{R}}_{2, c}^{81}
$$

pour $c=1, \ldots, 35$.

Il ne nous a pas été possible d'obtenir des distributions complètes pour les communes autres que Toulouse. Nous avons alors décidé de supposer que la variance du revenu dans chaque groupe, pour chaque année, était, pour chaque commune, celle que nous observions à Toulouse.

Soit

$$
\begin{aligned}
& \mathrm{V}_{1, c}=\mathrm{V}_{1,30} \\
& \mathrm{~V}_{2, c}=\mathrm{V}_{2,30}^{t}
\end{aligned} \quad t=80,81 ; c=1, \ldots, 35 .
$$

Les densités des distributions de revenu utilisées dans les estimations, pour chaque observation, ont été choisies log-normales, c'est-à-dire de la forme :

$$
\frac{1}{R \sigma \sqrt{2 \pi}} \exp \left(-(\log R-\mu)^{3 / 2} \sigma^{\prime}\right)
$$


où

$$
\left\{\begin{array}{l}
\mu=\log \overline{\mathbf{R}}-\frac{1}{2} \sigma^{2} \\
\sigma^{2}=\log \left(\frac{V}{\bar{R}^{2}}+1\right)
\end{array}\right.
$$

Il faut noter que les distributions fiscales que nous avons utilisées laissent de côté certains des foyers fiscaux qui, ayant un revenu trop faible pour être redevables de l'impôt sur le revenu des personnes physiques, compte tenu du nombre de parts fiscales du foyer, ne déclarent pas celui-ci.

Cela entraîne donc un biais dans les distributions du revenu que nous avons utilisées : il manque des effectifs dans les premières tranches de revenu.

Dans notre échantillon, $\overline{\mathbf{R}}_{1 k}$ varie, suivant $k$, de $44262 \mathrm{~F}$ à $109455 \mathrm{~F}$ et $\overline{\mathbf{R}}_{2 k}$ de $74372 \mathrm{~F}$ à $146477 \mathrm{~F}$.

2. Les proportions $f_{1 k}$ et $f_{2 k}$ de chaque groupe pour chaque observation ont été facilement obtenues à partir des statistiques fiscales ci-dessus. Dans notre échantillon $f_{1 k}$ varie, suivant $k$, entre 0,80 et 0,93 .

3. Les populations des communes sont ici les nombres de foyers fiscaux. Ces nombres nous ont été communiqués par l'administration fiscale. Ils varient entre 139 et 98464 pour les foyers fiscaux qui ne payent pas la taxe professionnelle et entre 18 et 11313 pour ceux qui la payent.

4. Les taux $h^{*}{ }_{k}$ de la taxe d'habitation nous ont été fournis par les services de la Trésorerie générale de la Haute-Garonne; $h^{\prime}{ }_{k}$ (avec l'impôt foncier bâti) a été calculé à partir des mêmes sources.

Dans notre échantillon, ils varient, suivant $k$, entre $2,81 \%$ et $16 \%$ pour $h^{*}{ }_{k}$ et entre $7,47 \%$ et $33,9 \%$ pour $h^{\prime}{ }_{k}$.

5. Les rapports $\left(\frac{T}{B}\right)_{k}$ ont été obtenus ainsi : $\frac{T}{B}=\frac{\text { Ressources communales autres que taxe d'habitation et taxe professionnelle }}{\text { (taxe d'habitation }+ \text { taxe professionnelle)/h}}$ $=\frac{h \text { (Total du budget }- \text { taxe d'habitation }- \text { taxe professionnelle) }}{\text { taxe d'habitation }+ \text { taxe professionnelle }}$

Les données nécessaires au calcul figurent dans les comptes administratifs des communes.

Dans notre échantillon, $\left(\frac{T}{\mathrm{~B}}\right)_{k}$ varie, suivant $k$, de 0,0651 à 0,857 (variante $A$ ). Pour la variante $B$, le rapport analogue varie de 0,056 à 0,831 . 
6. Les coefficients $\delta_{k}$ ont été obtenus ainsi :

$$
\text { pour un agent, } \delta=\frac{t \times \text { Base taxe professionnelle }}{h \times \text { revenu }} .
$$

Nous avons considéré que tous les agents d'une même collectivité avaient la même valeur de $\delta$.

Soit $\delta_{k}=\frac{t_{k}}{h_{k}} \times$ base totale de la taxe professionnelle $\times \frac{1}{\mathbf{N}_{2 k} \widetilde{\mathbf{R}}_{2 k}}$ où $\mathbf{N}_{2 k}$ est le nombre total des foyers fiscaux du groupe qui paye la taxe professionnelle et $\overline{\mathbf{R}}_{2 k}$ est le revenu moyen du groupe.

Dans notre échantillon, $\delta_{k}$ varie, suivant $k$, de 0,055 à 0,937 pour la variante $A$ et de 0,003 à 0,355 pour la variante $B$.

\section{LES RÉSULTATS}

Nous procédons tout d'abord à un ensemble d'estimations en incluant dans le budget communal qui fait l'objet du vote uniquement la taxe d'habitation. Nous montrons comment, conformément à la théorie, la précision des estimations s'améliore lorsqu'on passe successivement d'une estimation par les DMCNL avec des instruments quelconques, à une estimation par les doubles moindres carrés non linéaires avec des instruments optimisés (notée DMCNLO) et enfin à une estimation par la méthode du maximum de vraisemblance (notée MV).

Diverses tentatives ont été réalisées pour analyser une éventuelle hétéroscédasticité des erreurs $\varepsilon_{k}$ dans l'équation (16). Toutefois, comme les meilleurs résultats ont été obtenus dans le cas d'homoscédasticité (c'est-à-dire $q_{k}=1$ ), nous ne donnons ci-après que les estimations obtenues dans ce cadre.

Comme le problème est homogène en $\alpha, \beta, \gamma$, nous le normalisons en choisissant $\beta=0,5$.

Pour la première méthode, nous utilisons comme matrice d'instruments $W$, la matrice de rang 3 , dont la transposée s'écrit :

$$
\left[I_{3}, I_{3}, \ldots, I_{3} I_{2}\right]
$$

où $I_{l}$ désigne la matrice identité d'ordre $l$.

Les résultats suivants sont robustes à la méthode d'estimation : la fonction d'utilité incorpore une désirabilité des biens publics décroissante avec le revenu (l'estimation de $a$ est très stable). L'hypothèse démocratique, $v=1$, est rejetée au niveau $1 \%$. 
Tableau 1

\begin{tabular}{cccc} 
& DMCNL & DMCNLO & MV \\
\hline$\alpha$ & 18,09 & $\begin{array}{c}63,21 \\
(7,0)\end{array}$ & $\begin{array}{c}17,08 \\
(7,6)\end{array}$ \\
\hline$a$ & $(1,2)$ & $-0,009$ & $-0,016$ \\
& $-0,011$ & $(0,6)$ & $(13,5)$ \\
$v$ & $(1,9)$ & 13,29 & 6,03 \\
& 11,92 & $(3,4)$ & $(5,1)$ \\
\hline
\end{tabular}

- Lee nombres entre parentheses cont les retlos do Student.

L'hypothèse de normalité des erreurs est acceptée par un test de Kolmogorov-Smirnov (niveau de signification empirique 0,07); nous nnivons donc nous attacher aux estimations du MV. On s'aperçoit alors les agents qui payent la taxe professionnelle ont un poids trois fois ; important que les autres dans la décision collective. Le coefficient $\alpha$ est bien déterminé mais le coefficient $\beta$ est trop faible si l'on souhaite interpréter $\beta / \alpha+\beta$ comme la part du revenu consacrée au logement. Toutefois, cette interprétation n'est pas correcte car pour tous les propriétaires il faudrait rectifier le revenu en y imputant les loyers. Il n'est donc pas étonnant que $\beta$ soit sous-estimé fortement.

\section{ÉTUDES DE ROBUSTESSE}

Dans cette section, nous procédons à diverses variantes pour apprécier la robustesse des conclusions données à la section précédente. Nous utilisons uniquement la méthode du MV puisque l'hypothèse de normalité a été acceptée.

1. Nous incluons ici l'impôt foncier bâti dans le budget qui fait l'objet du vote (voir tableau 2). Les conclusions de la section 4 sont préservées.

Tableau 2

\begin{tabular}{|c|c|}
\hline $\boldsymbol{\alpha}$ & $\begin{array}{l}16,97 \\
(6,8)\end{array}$ \\
\hline$a$ & $T_{(12,1)}^{0,016}$ \\
\hline$v$ & $\begin{array}{c}9,79 \\
(3,34)\end{array}$ \\
\hline
\end{tabular}


2. En éliminant les communes de taille élevée, nous nous apercevons d'une chute très importante de $v$. Ceci nous amène à formuler le modèle de décision collective avec $v$ fonction de la taille de la population $\mathbf{N}$.

$$
v=v_{0}+v_{1} \frac{\log N}{\log N_{\max }}
$$

où $\mathrm{N}_{\max }$ est la taille de la population de Toulouse.

Tableau 3

\begin{tabular}{ccc}
\hline & $\begin{array}{c}\text { MV } \\
\text { sans } \\
\text { foncier bâti }\end{array}$ & $\begin{array}{c}\text { MV } \\
\text { avec foncier bâti }\end{array}$ \\
\hline \multirow{2}{*}{$a$} & 17,06 & 16,5 \\
& $(7,6)$ & $(6,4)$ \\
& $-0,016$ & 0,015 \\
$v_{0}$ & $(13,4)$ & $(11,6)$ \\
& 8,2 & 23,04 \\
$v_{1}$ & $(1,6)$ & $(2,1)$ \\
& $-3,48$ & 19,37 \\
& $(0,5)$ & $(1,5)$ \\
\hline
\end{tabular}

Le poids $v$ décroît avec la taille de la commune mais de façon peu significative.

3. Deux soucis majeurs subsistent. Le mode de calcul que nous avons retenu pour la taxe professionnelle repose sur l'utilisation du coefficient $\delta$. Pour étudier la sensibilité des résultats, nous avons éliminé les cinq communes qui ont un $\delta$ élevé, c'est-à-dire une part de la taxe professionnelle forte dans le budget communal. Ceci ne perturbe pas sensiblement les résultats et rend $v$ plus sensible à la taille de la commune (voir tableaux 4 et 5).

Enfin, la sous-évaluation des loyers utilisée pour le calcul des impôts locaux suggère que $h$ est surévalué. Diverses estimations en réduisant $h$ uniformément ne modifient pas les résultats.

Tableau 4

\begin{tabular}{ccc}
\hline & $\begin{array}{c}\text { MV } \\
\text { sans foncier bâti }\end{array}$ & $\begin{array}{c}\text { MV } \\
\text { foncier báti }\end{array}$ \\
\hline$\alpha$ & 10,6 & 15,7 \\
& $(7,4)$ & $(5,2)$ \\
$a$ & $-0,015$ & 0,015 \\
& $(9,4)$ & $(9,3)$ \\
0 & 12,66 & 15,43 \\
& $(3,0)$ & $(2,4)$ \\
\hline
\end{tabular}


Tableau 5

\begin{tabular}{lcc}
\hline & $\begin{array}{c}\text { MV } \\
\text { soncier bâti }\end{array}$ & $\begin{array}{c}\text { MV } \\
\text { savec foncier bati }\end{array}$ \\
\hline$\alpha$ & 8,9 & 14,6 \\
$(4,4)$ & $(5,9)$ \\
$a$ & 0,015 & 0,014 \\
& $(9,5)$ & $(8,2)$ \\
$v_{0}$ & 44,17 & 56,25 \\
& $(2,0)$ & $(1,8)$ \\
$v_{1}$ & $-42,86$ & $-54,71$ \\
\hline
\end{tabular}

\section{CONCLUSION}

Bien qu'il soit prématuré de tirer des conclusions d'une étude reposant sur une base de données aussi limitée que la nôtre, nous sommes amenés à formuler deux hypothèses qui devront faire l'objet de tests plus approfondis.

D'une part, le désir de bien public semble corrélé négativement avec le revenu. Il faut noter toutefois que nos hypothèses a priori sur les préférences sont très fortes. D'autre part, l'hypothèse démocratique est rejetée et ceci d'autant plus que les communes sont petites. Il existe au moins deux raisons pour un tel résultat. Des communes cherchent à attirer les agents qui payent la taxe professionnelle et donc sont très attentives à leurs désirs. Il est bien connu que les campagnes électorales se font chez les commerçants.

Nous espérons pouvoir approfondir prochainement ces conjectures avec une base de données plus importante. 
ANNEXE

\section{VRAISEMBLANCE ET VRAISEMBLANCE CONCENTRÉE}

D'après (16) nous avons, pour une observation :

$$
g\left(h^{*},\left(\frac{T}{B}\right), \delta, \overline{\mathbf{R}}_{1}, \overline{\mathrm{R}}_{2}, \sigma_{1}, \sigma_{2} ; \alpha, \beta, \alpha, v\right)=\varepsilon
$$

Par hypothèse, $\varepsilon \sim \mathscr{N}\left(0, \sigma^{\circ}\right)$, donc la densité de $h^{*}$ est :

$$
\frac{1}{\sqrt{2 I I} \sigma} \exp \left\{-\frac{1}{2 \sigma^{2}} g\left(h^{*}, \ldots\right)^{2}\right\} \cdot\left|\frac{\partial g}{\partial h^{*}}\right|
$$

Notons $\mathrm{J}_{k}$ la valeur de $\left|\frac{\partial g}{\partial h}\right|$ pour la commune $k$.

Le logarithme de la vraisemblance s'écrit :

$$
L=-\frac{K}{2} \log \left(2 I I \sigma^{2}\right)-\frac{1}{2 \sigma^{2}} \sum_{k} \varepsilon_{k}^{2}+\sum_{k} \log J_{k}
$$

On concentre la vraisemblance en optimisant par rapport à $\sigma^{2}$ :

$$
\frac{\partial \mathrm{L}}{\partial \sigma^{2}}=-\frac{\mathbf{K}}{2 \sigma^{2}}+\frac{1}{2 \sigma^{2}} \sum_{k} \varepsilon_{k}^{2}
$$

et

$$
\partial^{2}=\frac{1}{K} \sum \varepsilon_{k}^{2}
$$

Reportant ceci dans la log-vraisemblance, nous obtenons la logvraisemblance concentrée :

$$
L_{c}=-\frac{K}{2} \log (2 \text { II } \partial y)-\frac{K}{2}+\sum_{k} \log J_{k}
$$

C'est l'opposée de cette fonction qui a été minimisée pour l'estimation par Maximum de Vraisemblance.

Calcul de $\frac{\partial g}{\partial h^{*}}$ :

$$
\frac{\partial g}{\partial h^{*}}=\frac{f_{1}}{f_{1}+\partial f_{2}} \frac{\partial \Phi}{\partial h^{*}}\left(b_{1}\right)+\frac{\partial f_{2}}{f_{1}+\partial f_{2}} \frac{\partial \Phi}{\partial h^{*}}\left(b_{2}\right)
$$

où

$$
b_{i}=\frac{\log R_{i}-M_{i}}{\sigma_{i}} \quad i=1,2
$$


$\Phi$ est la fonction de répartition de la loi $\mathscr{N}(0,1)$.

$$
R_{1}=\frac{1}{a \cdot c t e} \log \gamma_{1} \quad R_{2}=\frac{1}{a \cdot c t e} \log \gamma_{2}
$$

où $\operatorname{ct} e\left(=10^{-3}\right)$ est une constante de normalisation.

$$
\begin{gathered}
\gamma_{1}=\beta \cdot\left(\frac{h^{*}+\left(\frac{T}{B}\right)}{1+h^{*}}\right) \\
\gamma_{2}=\gamma_{1}+(\alpha+\beta) \cdot \delta \cdot\left(\frac{h^{*}+\left(\frac{T}{B}\right)}{1-\delta \cdot h^{*}}\right)
\end{gathered}
$$

Notons $\varphi$ la densite $\mathscr{N}(0,1)$. Alors :

$$
\begin{gathered}
\frac{\partial \Phi\left(b_{i}\right)}{\partial h^{*}}=\varphi\left(b_{i}\right) \cdot \frac{\partial b_{i}}{\partial h^{*}}=\varphi\left(b_{i}\right) \cdot \frac{1}{\sigma_{i} \mathbf{R}_{i}} \cdot \frac{\partial \mathbf{R}_{i}}{\partial h^{*}} \quad i=1,2 \\
\frac{\partial \mathbf{R}_{1}}{\partial h^{*}}=\frac{1}{a \cdot c t e \cdot \gamma_{1}} \cdot \beta\left(\frac{1-\left(\frac{T}{B}\right)}{\left(1+h^{*}\right)^{2}}\right) \\
\frac{\partial \mathbf{R}_{2}}{\partial h^{*}}=\frac{1}{a \cdot c t e \cdot \gamma_{2}} \cdot \beta\left\{\frac{1-\left(\frac{T}{B}\right)}{\left(1+h^{*}\right)^{2}}+(\alpha+\beta) \cdot \delta \cdot \frac{1+\delta\left(\frac{T}{B}\right)}{\left(1-\delta h^{*}\right)^{2}}\right\}
\end{gathered}
$$

En reportant ces expressions dans (a. 3) on obtient le jacobien.

\section{RÉFÉRENCES BIBLIOGRAPHIQUES}

Amemrya T. [1975], - The non linear two-stage least squares estimator $"$ Journal of Econometrics, 2, p. 105-110.

Bercstrom T., Goodman R. [1973], * Private demands for public goods ", American Economic Review, 63, p. 280-296.

Comanon W. [1976], "The median voter rule and the theory of political choice *, Journal of Public Economics, 5, p. 169-177.

Gallunt A. R., Jongenson D.W. [1979], a Statistical inference for a system of simultaneous, non-linear, implicit equations in the context of instrumental variable estimation ", Journal of Econometrics, 11, p. 275-302.

Romera T., Rosentrax. H. [1979], * The elusive median voter ", Journal of Public Economics, 12, p. 143-170. 